\title{
Primary lliopsoas Abscess in a Neonate
}

\author{
Muhammad Umar Nisar1, Samer Sikander33, Zenab Noorain², Mehr-un-Nisa Shahid Baig1 and Nadeem Akhtar ${ }^{1}$
}

\begin{abstract}
Iliopsoas abscess (IPA) is rare in children but exceptional in neonates. Fewer than 30 cases have been reported in literature. We present a case of 11-day neonate who was brought with left inguinal swelling along with significantly raised white cell count and positive C-reactive protein (CRP). Ultrasound and CT scan confirmed the diagnosis of IPA. Drainage of abscess was done by open method through extraperitoneal approach. Systemic antibiotics, according to culture and sensitivity, were given and the neonate recovered well and was sent home. IPA can be primary or secondary. Primary IPA is more common in neonates unlike adults, who have secondary IPA in majority of the cases. Primary IPA spreads by hematogenous route from distant occult source in the body. Staphylococcus aureus is the causative organism in most cases of primary IPA. Septic arthritis of hip joint is among important differentials. Ultrasound and CT scan are helpful in diagnosis in the presence of raised white cell count and positive CRP. Drainage of pus by open method and through extraperitoneal approach is preferred method although ultrasound-guided percutaneous drainage has also been done with successful outcome. In conclusion, neonatal IPA is extremely rare entity and can easily be overlooked. High index of suspicion is required for its diagnosis in cases where a neonate presents with groin swelling, limited or painful motion of leg and fever.
\end{abstract}

Key Words: Iliopsoas abscess, Neonate, Idiopathic, Pakistan.

\section{INTRODUCTION}

lliopsoas abscess (IPA) is not a common disease in children and it is considered exceptional in neonates. First description of IPA was given by Mynter in 1881 and was called "psoitis". ${ }^{1}$ Fewer than 30 cases of neonatal iliopsoas abscess have been reported in literature so far. ${ }^{2}$ To the authors' knowledge, this is first case report from Pakistan. The authors present exceptional case of IPA in a 11-day old neonate who presented to us with complaints of reluctance to feed, swelling around left hip joint, crying while changing diaper and fever. IPA was diagnosed on ultrasonography and computed tomography. Incision and drainage of abscess was done and around $30 \mathrm{cc}$ of pus was drained. The neonate was given intravenous antibiotics, according to culture and sensitivity, and was discharged when stable and tolerating oral feed.

\section{CASE REPORT}

A full-term female neonate with birth weight of $2.3 \mathrm{Kg}$ was born by caesarean section due to maternal history of short pelvis. The pregnancy and post-natal period

\footnotetext{
1 Department of Paediatric Surgery / General Surgery ${ }^{2}$, The Children's Hospital, Pakistan Institute of Medical Sciences, Islamabad, Pakistan

3 Department of Paediatric Medicine, PAEC General Hospital, Islamabad, Pakistan

Correspondence: Dr. Muhammad Umar Nisar, Department of Paediatric Surgery, The Children's Hospital, Pakistan

Institute of Medical Sciences, Islamabad, Pakistan

E-mail:dr.umarnisar@yahoo.com

Received: November 14, 2018; Accepted: January 02, 2019
}

were uneventful and the neonate was discharged on full breast feed.

On $11^{\text {th }}$ day of life, swelling was noted in the left hip region with excessive crying on changing diaper. She was brought to the pediatric emergency department on the next day with complain of fever and refusal to take feed .BCG vaccination was already given on the 3 rd day of life. The neonate did not receive any intra-muscular injection. There was no history of trauma or any insect bite on the affected thigh.

On examination, she looked unwell and there was illdefined swelling in left hip region extending upto the left flank, with bluish discoloration of the surrounding area (Figure 1). She had abduction deformity of left leg. There was limitation of range of motion. Oral thrush was also noted although the neonate was exclusively breast-fed.

Laboratory investigations revealed haemoglobin of $10.3 \mathrm{gm} / \mathrm{dL}$, WBC count of $42.9 \times 10^{9} / \mathrm{L}$, platelet count of $825 \times 10^{9}$, neutrophil count $73.8 \%$ and CRP was $96 \mathrm{~g} / \mathrm{L}$. Her serology tests were negative and blood culture was positive for pseudomonas aeruginosa. Chest X-ray and $\mathrm{X}$-ray abdomen pelvis including hip joints were normal. Bone scan was also done and no bony pathology was noted. Ultrasonography revealed hypoechoic areas with thick internal echoes measuring approximately $14 \mathrm{ml}$ involving left illiopsoas muscle extending into the gluteal region and proximal part of thigh suggestive of abscess formation. CT-scan pelvis with contrast showed left IPA extending from the level of iliac crest down to left thigh with few enlarged inguinal lymph nodes with surrounding soft tissue edema of upper thigh and buttocks (Figure 2). 


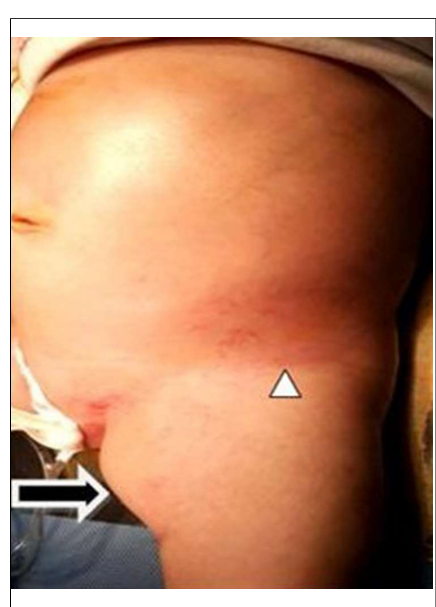

Figure 1: Swelling in left inguinal and upper thigh (black arrow) and redness in left inguinal region (arrow head).

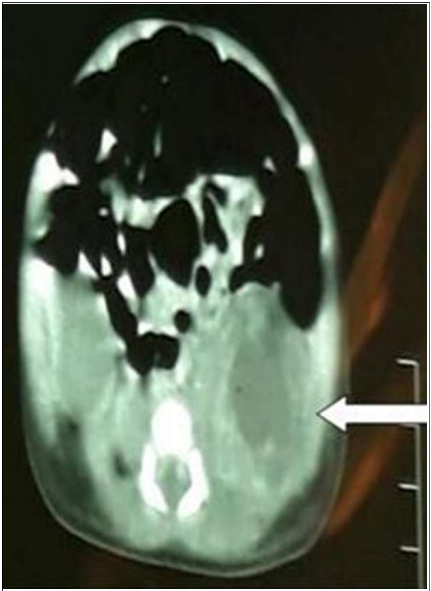

Figure 2: CT scan showing collection in the region of iliopsoas muscle. (white arrow).
Incision and drainage through extraperitoneal approach was done and about $30 \mathrm{cc}$ of frank pus was drained. Pus culture yielded growth of methicillin resistant staphylococcus aureus (MRSA) after 24 hours of incubation, which was sensitive to linezolid.

She was given intravenous systemic antibiotic therapy for one week and was discharged home in good condition and on full feed.

\section{DISCUSSION}

lliopsoas abscess (IPA) can be primary or secondary. Primary IPA occurs more likely due to hematogeneous spread from distant occult source of infection in the body because of weak immunity in neonates. This is aided by the fact that in majority of cases of primary IPA, the etiologic agent was staphylococcus aureus, which is commensal flora of skin. Secondary IPA is a result of direct extension of infection from adjacent structures into the iliopsoas muscle. Secondary IPA is more common in adults and the most common causes are intraabdominal inflamatory processes, particulary of intestinal origin . Primary IPA accounts for the majority of cases in neonates. ${ }^{1,3}$

IPA in neonates can easily be overlooked because of its rarity; and high index of suspicion is required for its diagnosis. Most common symptoms reported in literature are triad of swelling in femoral triangle, reduced movements of leg and fever. ${ }^{3}$ Other symptoms may include reluctance to feed and excessive crying.

The most important differential diagnosis of IPA is septic arthritis of hip joint. As in this case, it was one of differentials, so bone scan done to rule out any inflamatory changes in hip joint. One case of IPA reported in literature, was reffered to pediatirc surgery as a case of inguinal hernia. ${ }^{4}$ Other differentials include lymphangioma, neoplasm, osteomyelitis and thrombosis. ${ }^{2}$
Routine hematological investigations such as complete blood count (CBC) and CRP aid in confirming inflamatory nature of swelling or mass. WBC count in cases of IPA can be increased utpo $30 \times 10^{9} / \mathrm{L}$; but in this case, it was $42.9 \times 10^{9} / \mathrm{L}$ and CRP was $96 \mathrm{~g} / \mathrm{L}$. Xray of the hip joint can be done to rule out septic arthritis. Blood cultures and aspirate cultures from abscess are, however, more accurate diagnostic tools for IPA with abcess aspirate being more specific. ${ }^{4}$

Ultrasound is non-invasive and easily availble modality that can be done on bedside and gives findings that are suggestive of abscess. High resolution ultrasound is also helpful in distinguishing arthritis from psoas abscess by revealing joint effusion in case of arthritis and collection in cases of IPA. Howerver, CT scan is helpful in giving accurate and deltailed information regarding extent of swelling and its volume. ${ }^{5} \mathrm{In}$ this case, ultrasound was showing hypoechoic areas with thick internal echoes measuring approximately $14 \mathrm{ml}$ involving left illiopsoas muscle and extending upto the gluteal region and proximal part of thigh, suggestive of abscess collection. CT scan confimed the diagnosis of IPA.

Staphylococcus aureus is the most common organism isolated in IPA. In $80 \%$ of primary IPA, S. areus has been reported as a causative agent. ${ }^{6}$ Eleven out of 13 cases of neonatal IPA, reviewed by Saikiran et al., had $S$. aureus as etiologic agent. ${ }^{7}$ Other organisms include Staphylococcus hominis, Klebsiella pneumoniae, and Streptococcus pneumoniae. ${ }^{3,4,7}$

Regarding treatment, review of literature showed that most of these patients were given emperical intravenous antibiotics due to high WBC count and positive CRP. In cases of multi-locutated abscess, open drainage by extraperitoneal approach is preferred. ${ }^{3}$ However, ultrasoundguided aspiration of abscess has also been reported in few cases with successful outcome. 8

Neonatal IPA is an extremely rare entity and can easliy be overlooked. High index of suspicion is required for its diagnosis in cases where a neonate presents with groin swelling, limited or painful motion of leg and fever.

\section{REFERENCES}

1. Al-Zaiem MM, Bajuifer SJ, Fattani MO, Al-Zaiem FM. Bilateral iliopsoas abscess associated with right hip septic arthritis in a neonate. Saudi Med J 2014; 35:743-6.

2. Smedegaard H, Heiring C, Hindsø K, Gormsen M, Teilmann G. Hip and thigh swelling at age 20 days. Neo Rev 2015; 16:e664-e7.

3. Duminda H, Lamahewage A, Liyange A, Fernando D. lliopsoas abscess due to methicillin resistant staphylococcus aureus in a 26-day-old neonate. Sri Lanka J Child Health 2015; 44:167-8.

4. Han YM, Kim AY, Lim RK, Park KH, Byun SY, Kim SH, et al. Neonatal iliopsoas abscess: The first Korean case. J Korean Med Sci 2015; 30:1203-6. 
5. Horiuchi A, Kameoka K, Kuwabara J, Watanabe Y, Kawakami S, Tauchi H, et al. Neonatal iliopsoas abscess. Pediatr Int 2012; 54:712-4.

6. Karabayir N, Turel O, Aydogmus C, Hatipoglu N, Hocaoglu A, Adal $\mathrm{E}$. Iliopsoas abscess in the neonate with immunodeficiency. Pediatr Int 2012; 54:439-40.
7. Saikiran D, Baswaraj T. Case 2: Groin swelling and fever in a 21-day-old girl. Neo Rev 2015; 16:e183-e4.

8. Dib M, Bedu A, Garel C, Mazda K, Philippe-Chomette P, Rajguru $\mathrm{M}$, et al. lliopsoas abscess in neonates: Treatment by ultrasound-guided percutaneous drainage. Pediatr Radiol 2000; 30:677-80.

-...…… 